\title{
Introducción: A 120 años del Natalicio de Lev Semiónovich Vygotski
}

\author{
Introduction: \\ 120 years of the birth of Lev Semyonovich Vygotsky
}

\author{
Adolfo Perinat Maceres \\ Universitat Autònoma de Barcelona, España
}

\begin{abstract}
"Celebremos la memoria de los grandes hombres que con sus ideas han sentado los cimientos de la humanidad" podría ser la divisa que enmarca este (y otros) homenajes a Vygotski, una de las figuras señeras de la psicología actual. No fue un científico cuya obra tuviera trascendencia en los días de su vida, ni en Rusia su país natal ni en Europa o América. Se le rescató del olvido 40 años más tarde de su muerte (1934). No sé si se ha estudiado con suficiente detalle los motivos de su redescubrimiento, por qué sucedió en un momento oportuno. Indudablemente algo tuvo que ver la incomodidad que Piaget y su extremo biologismo causaba en el colectivo de Psicólogos del Desarrollo infantil. Vygotski con sus ideas de la preeminencia de lo social en el desarrollo enarbolaba la bandera de los que creían que ya era el momento de reivindicar el peso de la socialización en el desarrollo del niño. Asunto afín es indagar si las primeras versiones de aquellas ideas no fueron una selección sesgada de un pensamiento que, a juzgar por investigaciones posteriores, es sumamente extenso, variado y no siempre coherente. Con esta apreciación aludo a una omisión fundamental: el marxismo en la psicología de Vygotski que fue vergonzosamente silenciado por parte de sus divulgadores americanos.
\end{abstract}

Desde los años 1970 y 1980 el fenómeno Vygotski no ha dejado de crecer y multiplicarse. El tema de la difusión de sus ideas en la comunidad científica es asimismo digno de estudiarse. Pienso que si el propio Lev Seminovich hubiera levantado la cabeza al final del siglo $X X$ se quedaría estupefacto de haberse convertido en una celebridad. Si Piaget ha dado de sí para un torrente de comentarios, réplicas de sus estudios infantiles y aplicaciones pedagógicas, Vygotski no ha quedado en zaga. (¿cuál es el próximo "gurú"?). Puede que ambos sean comparables en cuanto al interés suscitado y abundancia de comentaristas; puede que no lo sean tanto en lo que respecta a solidez epistemológica; pero este es otro asunto.

En mis primeros años docentes fui un fiel vygotskiano, devoto seguidor dentro de su masa de admiradores. No he dejado de citarle y de apoyar decididamente su postura pro-social, sin tampoco renegar de Piaget y su convincente interpretación biológica del desarrollo en Biologie et connaissance. Pero un día me sumergí a fondo en la lectura de Vygotski y en el análisis detallado de sus ideas. Me percaté que muchos de los citaban a Vygotski -por supuesto laudatoriamente- no se habían leído nada del personaje. O, si lo habían leído, carecían del mínimo sentido crítico. Muchas de las ideas de Vygotski no eran tan nuevas ni tan originales como lo habían pregonado sus admiradores. Puede que lo fueran en los años de 1930 -y no tengo motivos para dudar de ello- pero llegaban a la psicología del desarrollo en los años 1980, con un considerable desfase, y no aportaban demasiadas novedades. Su renombrada "teoría" histórico-cultural sienta la tesis - vertida a términos actuales- que la socialización es un producto de la acción social-comunicativa. ¡Esto ya estaba más que descubierto en los años finales de los 1970!. Por ejemplo, era patente en la obra colectiva de Martín Richards The Infant and his social World, (1974) donde Vygotski no aparece mencionado una sola vez.

Las reticencias prosiguen con las ideas de Vygotski acerca del signo: son confusas. Asume dos versiones: una es de aires pavlovianos, no solo por el asociacionismo que la preside y por considerarlo afín a un reflejo condicionado sino que, además, lo ampara bajo la analogía (pavloviana) del "tablero del telefonista" y otras concepciones obsoletas. Lo compensa subrayando certeramente el papel creador de la mente humana que lo hace esencialmente diferente de las señales del mundo animal y además extiende su papel al (auto) control de la conducta. La segunda versión es la del signo comunicativo, que surge ligado al lenguaje (a la palabra). Vygotski no establece explícitamente ninguna relación entre ambas modalidades de signos. En todo momento para Vygotski el signo es una conducta externa, lo cual dista mucho de ser cierto. La relación signo-símbolo nunca queda esclarecida (Vygotski no tuvo noticia de Peirce). Todo esto hace que su aproximación al signo y al símbolo sea bastante endeble.

El tercer gran "leit motiv" de Vygotski, el lenguaje, es más asumible. Su concepción del lenguaje interno como etapa de paso hacia el pensamiento (verbal) y el papel regulador de la palabra sobre la acción son 
dos aportaciones de gran nivel. En el tema del pensamiento no va más allá del ya indicado, el verbal; y en cuanto a sus ideas acerca del nacimiento del lenguaje sigue a la sombra de Pavlov y su rechazo visceral hacia la psicología idealista (una pesada herencia hegeliana) le impide reconocer que, en los niños, el progreso en el habla va de par con el del pensamiento. Sorprende también su distanciamiento de las corrientes lingüísticas de su tiempo, particularmente las rusas en plena efervescencia.

Que luego de este primer abordaje a fondo, mi impresión sobre Vygotski fuera un tanto desencantada tiene varias razones. Una de ellas nace de mis propias limitaciones: no he podido abordar su obra en su lengua original, el ruso, y la traducción española es bastante deficiente (para captarlo no hace falta compulsar el original...). Sus "obras escogidas" en español son una compilación desordenada, textos amontonados descuidadamente. Pero lo mas grave -y esto también es culpa mía- es que yo estaba leyendo a Vygotski a través de las categorías de la psicología de hoy. Su genialidad había de ser redescubierta a la luz de la psicología de los años de 1920-30: es la genialidad de una figura histórica. Sus ideas provienen en gran medida de la tradición filosófica y psicológica del siglo XIX: la dialéctica de Hegel, los postulados sociales en Marx, la psicología fisiológica de Pavlov, la Gestalt y los chimpancés de Köhler, la presencia absorbente y ubicua del doblete E - R, etc. Hay por tanto que encomiar la síntesis adecuada que intentó en aquel momento y que se tradujo en ideas muy avanzadas acerca del juego como "texto en acción", de la socialización a través de la comunicación, del habla organizadora de la actividad. La originalidad de Vygotski radica precisamente en haber esbozado (cosa que no logró enteramente) una síntesis entre la doctrina sociológica de Marx y la psicología a su alcance en la década de 1920-30. Estableció una carta de navegación que se alejaba por un lado de la corriente fisiológico-biológica y, por otro, de la que con Hegel se aferraba al ímpetu seminal del pensamiento (del "espíritu"). En Marx encontró su leit-motiv: la influencia de la sociedad en la construcción del hombre. Vygotski quiso darle un fundamento científico con su teoría histórico-cultural asimilando los signos a los instrumentos psicológicos que se emplean en esta construcción. En otras palabras, la analogía signo-instrumento da pie a Vygotski a elaborar una teoría que viene a ser la gran metáfora del marxismo: en paralelo al ascenso social de la humanidad mediante el trabajo y los útiles, Vygotski propone que la psique humana salta de los procesos elementales a los superiores gracias a la mediación del signo.

En cuanto a la psicología de Vygotski hay que decir que es un "collage" de Pavlov, la Gestalt, con aportaciones eventuales de Freud, inspiraciones darwinianas, conceptos que extrae de autores franceses Janet y Piaget y alemanes, Buhler, Stern, todos ellos citados en apoyo. No tiene unidad. El mismo lo reconoce en una especie de "grito existencial" que es su obra La crisis de la psicología. Evidentemente, no puede ser criticado por este eclecticismo de época. Bastante hizo con intentar abrirse paso en ese bosque de ideas y teorías que se encontró a mano. Pese a todo, la psicología de Vygotski tiene hondas raíces rusas. Isaiah Berlin dice que los pensadores rusos se imbuyeron del pensamiento europeo, siglo XIX, lo asimilaron y luego nos lo restituyeron filtrado por la cultura rusa de la época con las características sui generis que tuvo. Con ello no hago mas que abundar en la idea de que todas las corrientes o escuelas psicológicas están "situadas". La psicología fisiológica fue alemana y nació como reacción al idealismo hegeliano y las divagaciones poéticas de la Naturphilosophie. El holismo de la Gestalt era hijo de esas mismas grandes visiones omnicomprensivas de los alemanes del XIX. De Freud y las raíces vienesas y judías de sus elucubraciones, no digamos. El conductismo y la psicología del aprendizaje no podían ser sino norteamericanas con su empirismo dogmático y su ilusión de que "nada está grabado; el porvenir es fruto del esfuerzo individual". Mutatis mutandis, pienso que las ideas histórico-socio-culturales del Vygotski y el signo asimilado a una herramienta nacieron al socaire de las ideas de Marx asimiladas por la intelligentsia rusa revolucionaria y que él trató de trasladarlas al ámbito de la psicología. Sus ideas sobre el lenguaje interno son, en parte, de Sechenov; su principio de lo intermental-intramental es de Pierre Janet. Encuentra en Piaget la confirmación de que la reflexión infantil tiene un origen (social) en las discusiones entre amiguitos. Aunque al asumir estas ideas de la psicología de su tiempo, Vygotski les añade un toque personal, hay otras que acepta aun a riesgo de caer en incoherencias. Su posición, concretamente, hacia la reflexología es un buen ejemplo de ello. Puede que se deba a que Pavlov era un personaje "intocable" en la Rusia comunista y quizás por ello demuestra una clara ambivalencia frente al personaje; por otra parte, no tenía a mano otras de recambio porque la Gestalt y Freud, pese a que traían aires nuevos, no le convencieron. En síntesis, hay demasiadas piedras sillares nunca ajustadas en los cimientos de la psicología de Vygotski.

Esto afecta a la "actualidad de Vygotski" (titulo de un librito publicado hace algunos años en lengua española). No estoy muy de acuerdo que actualidad sea solo poner en circulación un haz de ideas suyas, cuidadosamente interpretadas, glosadas, incluso piadosamente depuradas sin entrar algo a fondo en sus fundamentos. Se ha elevado a primer plano la trascendencia de lo social y de la cultura en la formación de humanidad y del niño y el papel crucial del signo. Pero, su noción de cultura, la que maneja en su Historia del desarrollo de los procesos psicológicos superiores (Tomo III, Obras Escogidas) es ramplona. Resulta chocante de 
que luego de la explosión cultural en la Rusia de la primera década del siglo $\mathrm{XX}$ y de los movimientos de la organización post-revolucionaria de los años 1920, el Prolekult, y su concepción de una nueva cultura proletaria (arte, teatro, música, literatura, etc.) el lector crítico se tope con un Vygotski para quien la cultura era, cuando escribía ese texto, lectura, escritura, dibujo y aritmética. $\mathrm{O}$ sea, una concepción típicamente instruccional-escolar. Hacer pivotar sobre la cultura escolar toda la evolución psíquica de la humanidad (¡la cultura hace al ser humano!) es de una ingenuidad pasmosa. Como Vygotski no era un diletante, hay que buscar las razones de por qué reduce la cultura con " $\mathrm{C}$ " mayúscula a prácticas escolares (todo lo culturales que se quiera, pero escolares).

$\mathrm{Y}$ es que no se puede soslayar, estudiando a Vygotski, el panorama social y político de la Rusia de aquellos años y sobre todo la situación de los pensadores y literatos rusos (la "intelligentsia") en el régimen comunista (Vygotski murió en 1934 cuando se coronó el Primer Plan Quinquenal de Stalin). Los soviets, al menos sus jerifaltes, no eran unos incultos. Muchos de ellos habían formado parte de la intelectualidad zarista prerrevolucionaria. Eran conscientes de la influencia de los pensadores en Rusia. Suprimieron poco a poco las libertades de expresión, crearon un régimen de terror intelectual; luego, con Stalin, el terror de las ejecuciones o destierros a Siberia. La actividad científica se vio contaminada por la ideología sovieto-marxista. En las discusiones científicas se mezclaban indecentemente la crítica académica con la social: se trataba de "desenmascarar", "desvelar", "desmontar" las ideas que se desviaban de la línea del Partido. Vygotski fue objeto directo de una campaña de desprestigio: "Hemos analizado la "teoría histórico-cultural de la psicología" y prestado atención a los errores y distorsiones de los autores con respecto al marxismo. No hay duda de que los autores, Vygotski y Luria, son objetivamente partidarios de la influencia burguesa sobre el proletariado. No conociendo el marxismo, no utilizando el método del materialismo dialéctico, muestran en todo momento que se han apropiado de las directrices burguesas de moda en psicología, distorsionando y pervirtiendo el marxismo (Krementsov, 1997). Se trata del libro Studies on the History of Behavior: Ape, Primitive and Child, publicado en Rusia en 1931 (versión inglesa LEA, 1993).

En esta estremecedora atmósfera ¿quién, como Vygotski, podía dejar de citar a Marx y al Engels (a veces por compromiso), de jurar que la psicología había de ser marxista o dejar de existir (Crisis de la psicología), de rayar en el medioambientalismo al poner todo el peso de la construcción del psiquismo sobre la sociedad (sociogénesis), de elevar cantos de exaltación al trabajo como propulsor de la evolución de la humanidad, de saludar las primeras manipulaciones de los niños como "manejo de herramientas", de olvidarse de la esplendorosa cultura de élite rusa para refugiarse en la cultura escolar haciendo probablemente el juego (no sin convicción) a los grandes programas de educación popular soviéticos (alfabetización)? Et sic de ceteris. Tengo la certidumbre de que una gran parte de las ideas de Vygotski las expresa teniendo como trasfondo esta situación, típica de los regímenes totalitarios, que en Rusia alcanzó su paroxismo (aunque en la Alemania de Hitler no fue menos trágica).

Por todo ello, lejos de contentarse el estudioso con situar a Vygotski en el contexto de la psicología académica, ha de tener a la vista las circunstancias político-ideológicas en que se desenvuelve su corta vida. Un análisis únicamente textual, atemporal, es por si solo insuficiente. Vygotski vivió a caballo entre los estertores del antiguo régimen de los zares y los primeros años de la revolución proletaria comunista. Un tiempo atormentado, por una parte, pero, por otra, brillante en sus ideas y realizaciones culturales, antes e inmediatamente después de la Revolución (con Stalin la suerte cambió). El estudio de Vygotski sobre Hamlet puede tomarse como un reflejo de los aires de la época anterior a la avalancha comunista. Enseguida nos encontramos ante un Vygotski escindido en sus dos filias, la académico-occidental y la marxista. No pudo (o no quiso), a diferencia de Pavlov, dar la espalda a las presiones ideológicas. Todas estas son razones que nos retraen de abordar la psicología de Vygotski como si extrajéramos el significado de los conceptos que utiliza a partir de un diccionario de la disciplina. Siempre cabe preguntarse si su adhesión al marxismo fue sincera o fue oportunista. Infiero que participó del entusiasmo que contagió a los primeros comunistas por crear un mundo de relaciones sociales nuevo, diferente. Más concretamente, creo adivinar este propósito en la deriva de sus ideas hacia la educación, un asunto que Lenin tomó muy a pechos. Nunca sabremos qué sintió Vygotski ante estos terribles avatares de los que él mismo fue víctima; lo que sí creemos constatar es que asumió el marxismo científico con convicción y honestidad intelectual.

La herencia científica de Vygotski merece gran respeto; sobre todo nuevos aportes nacidos de la crítica y no solo glosas más o menos sustanciosas. Recojo una idea de George Steiner cuando sostiene que la critica puede auténticamente ser una re-visión, una interpretación, una glosa, una parodia del pensamiento de un clásico. ¿No son, dice, la Eneida de Virgilio y la Divina Comedia de Dante una relectura de Homero? Cada autor puede que inconscientemente traiga en sus episodios reminiscencias, ensoñaciones de los grandes predecesores, ecos de las epopeyas de la imaginación literaria. Extiende enseguida esta idea a la pintura ("el mejor crítico de Velázquez es Picasso"), a la música (las "Bachianas brasileiras" de Héctor Villalobos), etc. 
Quizás todo es una cadena sin fin... Me parece que esta sugerente propuesta es del todo aplicable a la obra de Vygotski. Puede que sus conceptos y recursos metodológicos fueran muy "primitivos", a medio camino entre el ensayo y una ciencia psicológica madura, pero pródigos en rasgos originales, puntos de mira novedosos, que han dado pábulo a comentarios, glosas, interpretaciones, ampliaciones y aproximaciones a autores coetáneos suyos (Bahktin, insospechadamente). Poco importa entonces que su teoría socio-cultural sea una reinterpretación de Marx (una "crítica" en el sentido que vengo comentando), que sus consideraciones sobre el signo vacilen entre Pavlov y los clásicos de "looking self-glass", que sus ideas sobre el lenguaje en relación con el pensamiento tengan un cierto olor museístico. Sí que nos importan las nuevas ideas brillantes a que han dado origen. Ha sido trascendental la resurrección de Vygotgsky en el área de la Psicología del Desarrollo para dar aires renovados a una atmósfera que por sus reminiscencias biológico-conductistas parecía una traslación de aquella a la que él se enfrentó.

Summa Psicológica ha tenido la laudable idea de sacar este número especial dedicado a Vygotski. Primero, tomo pie en el artículo de Alberto Labarrere y su clasificación de los comentaristas de nuestro personaje: los que entran en sus contenidos, su lógica, su pensamiento; los que buscan etapas en la evolución del mismo; los que extraen nuevas significaciones y tratan de construir sobre ellas. Quizás algo más simple sería la dicotomía entre los que entran a fondo en el pensamiento de Vygotski y los que se limitan a invocarle para refrendar sus trabajos. Otra clasificación es la que se puede establecer entre las indagaciones vygotskianas en psicólogos y pedagogos. Estos últimos han descubierto una veta inagotable en Vygotski para sus disquisiciones didáctico-misioneras. Todas las aportaciones en torno a, con pretexto de, Vygogtkii son bienvenidas. Sus méritos son relativos.

Alberto Labarrere y Rey González, van a la sustancia del pensamiento de Vygotski y tratan de explotar sus grandes potencialidades. Alberto Labarrere asume el desafío de decir algo sustancioso sobre la Zona de Desarrollo Próximo (en adelante ZDP), uno de los tema más manoseados de Vygotski y cuya originalidad no es deslumbrante. Labarrere ha hecho un análisis muy sagaz de la ZDP en condiciones de puesta en práctica -yo diría- ideales. Pero, aparte ese problema, su concepción personal (Vygotski, como él mismo dice, no entró en muchos detalles) contiene todos los ingredientes necesarios para una óptima interacción en la ZDP: hay una llamada insistente a la motivación en el aprendiz, a la intencionalidad compartida entre éste y el experto, hay una proyección necesaria hacia algo tan crucial como es "aprender a aprender" (metacognición). Me parecen muy oportunos sus puntos de vista sobre los SEAOD que, desafortunadamente, no son fáciles de implementar en situaciones de aprendizajes habituales, es decir, escolares. Porque este es "talón de Aquiles" de la ZDP de los pedagogos. Los que tenemos una experiencia docente aguerrida ( $y$ el que esto escribe la tiene de mas de 40 años) y hemos padecido las lacras de la cultura escolar, incluso universitaria, sabemos algo de ello.

Pero no quiero clausurar el tema de la ZDP sin añadir un punto de vista que, cada vez que lo he aireado, me ha atraído las protestas de los devotos de Vygotski. No encuentro ninguna originalidad en Vygotski en su enunciado de la ZDP. Cualquiera mínimamente familiar con los Diálogos de Platón, habrá advertido que su maestro, el viejo y astuto Sócrates, está constantemente actuando con sus interlocutores en la zona de su desarrollo próximo. Lean los devotos de Vygotski (si tienen humor para ello) La República, el Fedro o el Fedón y entérense que el territorio dialógico ha sido roturado y sembrado por los griegos ya desde el siglo $\mathrm{V}$ aC. No solo eso. El que esto escribe ha investigado a fondo y publicado sobre las interacciones primordiales madres-criaturas. Las mamás desde muy temprano están siempre actuando en la zona de desarrollo próximo de sus pequeños. Están alumbrando sus potencialidades porque saben que éstas existen, que los pequeñines están motivados para aceptar su "expertise" y que el desarrollo es convertir en acto lo que está en potencia. Todo esto está muy bien detallado por Labarrere; solo invito a cambiar el escenario.

González Rey centra su contribución en detallar tres momentos de la trayectoria intelectual de Vygotski. Seguramente es así aunque, como él mismo, reconoce, su obra es dispersa y -precisa- que no existieron en cada período ideas predominantes, como no sean las de la "teoría cultural". Con respecto a la Psicología del Arte, que González Rey encomia, tengo la impresión de que es una "rara avis" dentro del corpus vygotskiano. No veo claro cómo integrarla en él porque está mas relacionada con sus ideas estéticas que con las psicológicas. No es que unas y otras estén reñidas pero su engarce es dificultoso, ausente en Vygotski. Como muestra tenemos su abordaje del símbolo: no tiene ningún parentesco el que presenta en su ensayo sobre Hamlet y como más tarde lo trata en el dominio psicológico. Un símbolo es una forma de comunicación motivada y en la motivación siempre hay tintes emocionales y por aquí, como bien apunta González Rey, podrían tenderse puentes. En cuanto a la "teoría socio-cultural", segundo momento de la obra de Vygotski, ya he expresado arriba mi opinión. González Rey cita a autores rusos que la califican de mito. Yo convendría en ello pero quitando el matiz peyorativo y recuperando el original: mito es un relato. El tercer momento de Vygotski es el que, siguiendo sus comentaristas, abre perspectivas más novedosas y fecundas. Aquí entra en juego una conjunto de ideas que Gonzá- 
lez Rey enumera y comenta: conexión entre intelecto y afecto, pensamiento engastado en la motivación, pensamiento como función de sentido (¿sentido de qué?), sistema psíquico con capacidad autogeneradora y otras. Todo es muy seductor y auguro que se supere el gran problema de datar estas ideas dentro del "totum revolutum" de sus páginas. No hay posibilidad de descubrir etapas en el pensamiento de un autor si no se tiene una cronología aceptable de sus escritos.

Los siguiente trabajos corresponden aproximaciones empíricas vinculadas a algún concepto o idea Vygotskiana. La colaboración de Claudia González-Moreno, Yulia Solovieva y Luis Quintanar sobre la "adquisición de las acciones perceptivas simbólicas en niños preescolares" (Bogotá), retoma un tema que tiene mucha tradición en la psicología del desarrollo. Es aceptable que, como investigadores, quieran cerciorarse de que los niños colombianos transitan por el sendero universal de la infancia. No obstante, cabe destacar que una representación (gestual o gráfica o lo que sea) no es, de por sí, simbólica a menos que sea aceptada (convenida) por la sociedad. En otros términos, un símbolo es siempre una representación de algo (Peirce) pero no la recíproca. Sospecho que su noción de representación está acotada a la iconicidad; no sé que opinará al respecto Picasso. Cuando la nena de Piaget, Jacqueline, "se echa a dormir" no está haciendo (con perdón de su papá) ninguna acción simbólica sino representativa: está mimando. Cuando los niños de Bogotá hacen "garabatos" representando el enojo de su profesora, podrían -los garabatos- ser simbólicos si la sociedad conviniera en que representan ese enfado. ¿O qué otra cosa sino "garabatos" son las letras E-N-O-J-O y constituyen un símbolo? Todo esto para subrayar que la relación mutua representación-símbolo es compleja.

Finalmente el artículo de Ignacio Figueroa sobre el Rol Mediador de Aprendizajes en Educación Parvularia también se extiende en un campo que está muy roturado. El propósito del autor es contribuir a mostrar una línea de trabajo en Chile que muestra una aplicabilidad de la teoría vigotskyana. El artículo hace mención de una investigación en el marco de un programa de formación continua cuyo fundamento es de "matriz sociocultural, promoviendo la construcción reflexiva y colaborativa de herramientas pedagógicas que permitan fomentar el desarrollo cognitivo de los párvulos". Es de suponer que todos los programas de formación, sobre todo los escolares, tienen una matriz sociocultural. ¿Cómo puede ser de otra manera? Salirse de esta matriz sería un atrevimiento que la institución no puede permitirse ya que, a mi juicio, toda revolución en el ámbito de educación escolar está condenada de antemano. La reflexión, entonces, queda en manos de sus interlocutores.

Los artículos, colaboraciones diversas que aquí quedan expuestos, no hacen más iniciar su recorrido cuan- do salen a la luz en Summa Psicológica. Es de esperar y de desear que tengan un eco de crítica (aprobación, matices, objeciones, correcciones, etc.) y cumplan así su finalidad de publicación. No se siente exento de esa crítica, sino todo lo contrario, este presentador-colaborador de este número de Summa Psicológica, dedicado a Vygotski, al que deseo una buena acogida en su ámbito.

\section{Referencias}

Krementsov, N. (1997). Stalinist Science. Princeton University Press.

Vygotsky, L., Luria, A., Golod, V. \& Knox, J. (1993). Studies on the history of behavior: Ape, primitive, and child. Hillsdale: Lawrence Erlbaum Associates, Inc. 